\title{
THÈSES DE SOCIOLOGIE ET ROMANS À THÈSE
}

\author{
Michel Villette*
}

Résumé: Les marqueurs rhétoriques de la "scientificité" des discours sociologiques sont-ils spécifiques? Pour aborder cette question sensible, partons d'un tableau des procédés rhétoriques couramment employés en Sociologie. Déduisonsen les caractéristiques génériques du genre «écrit sociologique». Comparons ce genre à un genre littéraire à la fois proche et distinct: le roman à thèse (un genre mineur, très pratiqué en France au début du XXe siècle et illustré notamment par Charles Mauras et Paul Bourget). Les parallèles sont troublants. Mais alors, si la différence entre thèse de sociologie et roman à thèse n'est pas marquée dans le texte, ne faut-il pas chercher ailleurs la "scientificité", dans les fragiles conditions de la production et de la réception savante: débat sur l'interprétation et critique des sources? Encore faut-il que ces conditions se trouvent remplies, ce qui n'est pas si fréquent.

Mots-clés: Sociologie, roman à thèse, genre littéraire, rhétoriques, scientificité.

Une fois, on m'a invité à donner une conférence devant un vaste auditoire; elle devait commencer à vingt heures.

Schelling (1980, p. 17)

\footnotetext{
* Professeur de Sociologie à l'École Nationale Supérieure des Industries Alimentaires et à l'École Doctorale Entreprise, Travail, Emploi. Il a été cadre au groupe Danone, consultant à Eurequip, directeur d'études à l'Institut Entreprise et Personnel et professeur associé à l'ESCP. Il a publié L'Homme qui croyait au management (Seuil, 1988), L'Art du Stage en Entreprise (La Découverte, 1994, 1999), Le Manager Jetable (La Découverte, 1996). Ses travaux actuels portent sur les pratiques des hommes d'affaires. Adresses: ENSIA, 1 avenue des olympiades 91744 Massy Cedex. Courrier électronique: villette@ensia.inra.fr
}

Artigo recebido em 16 de mai. 2003; aprovado em 30 ago. 2003 
Sa femme (dont le père est gardien de la paix et la mère vendeuse dans un grand magasin) à 26 ans, elle est secrétaire à la Régie Renault depuis

cinq ans.

Bourdieu (1979, p. 384)

Par exemple, s'il suit l'étiquette, un homme ne devrait pas inviter trop tôt une jeune fille pour le réveillon du Nouvel An, de peur que celle-ci n'ait de la peine à trouver un prétexte honorable pour refuser.

Goffman (1974, p. 29)

Jusqu'à nos jours, l'empereur-pontife de Chine est resté un faiseur de pluie car, tout au moins dans la Chine du Nord, les incertitudes météorologiques revêtaient plus d'importance encore que les installations d'irrigation, aussi grand que soit le poids de celles-ci.

Weber (1971, p. 472)

Quel est le statut du récit dans les textes de sciences sociales? En quoi contribue-t-il à faire du discours sociologique un discours « scientifique »? Tantôt, il semble que la documentation empirique joue un simple rôle d'illustration des thèses avancées. Elle remplit alors la même fonction rhétorique que l'exemplum aristotélicien, la fable d'Esope ou la parabole évangélique. Tantôt, au contraire, les narrations, les extraits de conversation ou les dénombrements semblent être des résumés fidèles d'un compte rendu d'observation analogue au cahier d'expérience du biochimiste: à la fois source d'invention et élément de preuve, référent d'analyse issu d'une confrontation serrée et sérieuse avec le réel. On peut alors être tenté d'attribuer au texte un statut "scientifique" au sens où l'on n'hésite pas à employer ce terme, en dépit des équivoques qu'il recouvre, dans les sciences physiques ou les sciences de la vie (Latour \& Fabri, 1977; Latour \& Woolgar, 1979).

Considérez les descriptions empiriques (qu'elles soient chiffrées ou littéraires) comme des éléments ayant pour fonction la persuasion ou l'illustration pédagogique: la Sociologie devient aussitôt une branche de la rhétorique. L'art du sociologue est de faire advenir et de rendre crédible une représentation de la vie en société. Il emploie pour ce faire toutes sortes de moyens d'influence et, parmi ceux-ci, des procédés rhétoriques analogues à ceux des hommes politiques, des avocats, des prédicateurs et des auteurs de romans. 
Acceptez de considérer les mêmes descriptions comme des comptes rendus d'expérience fidèles, précis, exacts, complets, susceptibles de démentir telle ou telle théorie savante comme de l'illustrer; exigez la production de documents à l'appui, de preuves susceptibles de vérification, et la Sociologie devient une science empirique comme les autres, avec ses expériences, ses débats sur la différence entre faits et artefacts, ses fraudes, etc.

Pour montrer que la rhétorique d'exposition des résultats de recherche (dans des articles ou des livres) est insuffisante en ellemême pour caractériser comme « scientifique » le texte du sociologue, (et plus sérieusement, pour suggérer que la transgression raisonnée de certains aspects de cette rhétorique serait parfois de nature à accroître la scientificité du propos), on s'est attaché ici à comparer deux genres dont l'un relève de la littérature et l'autre de la science: le roman à thèse et l'article de Sociologie.

On part d'une grille de lecture des procédés rhétoriques couramment employés dans la littérature sociologique standard proposée par Joe Gusfield (1987). On en déduit un ensemble de caractéristiques génériques du genre «écrit sociologique ». On compare ces caractéristiques avec celles d'un genre littéraire à la fois proche et distinct: le roman à thèse, tel qu'il a été analysé par Suzanne Rubin Suleiman (1983). Ce genre littéraire fort décrié sert en quelque sorte de repoussoir ${ }^{1}$ pour mettre en évidence des procédés parfois revendiqués comme "scientifiques" bien qu'ils ne soient pas spécifiques du champ savant et qu'ils n'apportent pas, en eux-mêmes, la scientificité recherchée.

Une des difficultés constantes de l'analyse rhétorique d'un genre littéraire est la délimitation des frontières de celui-ci. Je propose d'éluder cette difficulté: les genres "thèses de sociologie" et "roman à thèse" seront construits par référence à quelques textes typiques. Il incombe au lecteur d'appliquer la grille d'analyse à tel ou tel texte particulier pour décider de sa proximité plus ou moins grande aux modèles de référence proposés. 
L'analyse de ce que Joe Gusfield appelle "la littérature sociologique standard américaine" s'appuie sur le dépouillement d'un grand nombre d'articles, de rapports et de livres qui peuvent être groupés en trois sous-genres: les essais théoriques et de synthèse (exclus de notre analyse), les enquêtes à base de données statistiques (statisticals inquieries) et les monographies à base de données qualitatives (field studies). Pour illustrer son propos, Gusfield s'appuie sur la comparaison entre deux classiques de la sociologie américaine: The American Occupational Structure, de Peter M. Blau et Otis D. Ducan (1967), et Street Corner Society, de William Foote Whyte (1943) représentant respectivement l'enquête statistique et la monographie de terrain.

L'analyse de Joe Gusfield conduit à une étude contrastée des sous-genres à l'intérieur du domaine de la sociologie. Elle débouche aussi sur une liste de caractéristiques formelles communes à l'ensemble des productions "standards" de la discipline, encore très pertinente aujourd'hui.

Susan Rubin Suleiman dans Le Roman à Thèse, analyse les caractéristiques formelles de romans comme Les Déracinés de Maurice Barrès, L'Étape de Paul Bourget, La Conspiration de Paul Nizan, L'Enfance d'un Chef de Jean-Paul Sartre. Elle part d'une définition signalétique du roman à thèse ${ }^{2}$ comme "un roman réaliste" (fondé sur une esthétique du vraisemblable et de la représentation) qui se signale au lecteur principalement comme porteur d'un enseignement, tendant à démontrer la vérité d'une doctrine politique, philosophique, scientifique ou religieuse" (Suleiman, 1983, p.14).

Faisons donc l'hypothèse qu'écrits sociologiques standards et roman à thèse sont deux genres littéraires qui partagent beaucoup de caractéristiques communes. Pour éprouver les limites de cette hypothèse, partons de la liste des caractéristiques du texte sociologique standard définie par Joe Gusfield et cherchons à repérer, pour chaque caractéristique, son équivalent dans le roman à thèse: 


\section{Dans son texte, le sociologue s'efface en tant que personne et prend de la distance vis à vis de son sujet}

Selon Joe Gusfield, le premier caractère formel typique de l'écrit sociologique standard est l'absence de la première personne du singulier et l'utilisation conventionnelle du « nous » (parfois remplacé en français par la tournure impersonnelle «on» pour désigner l'énonciateur.

Ce n'est pas le sociologue, en tant que personne singulière, qui s'exprime mais un « sujet savant ». Un ensemble de formules signalent la distance (ou l'extériorité) de ce sujet savant par rapport au champ social qu'il étudie: «On a observé que ...»; «les membres de l'entreprise disent que ... ». Distinct de la personne sociale de l'auteur (avec ses « opinions personnelles », sentiments moraux et préférences politiques), l'auteur sociologique se distingue aussi de ceux dont il parle et avec lesquels il tend à n'avoir rien de commun, comme pour rester impartial. L'énonciateur d'un texte sociologique n'est pas pour autant un locuteur indéterminé, bien au contraire.

L'appartenance à une institution de recherche reconnue, la liste des travaux et publications, les remerciements aux collègues et bailleurs de fonds, les citations affirment à priori la légitimité scientifique de l'auteur et marquent la différence de nature entre ce qu'il peut dire et ce que pourraient dire des profanes sur le même sujet. Surtout, ces références créent un réseau intertextuel dans lequel s'insère l'écrit sociologique et par référence auquel il prend sens. Supposées connues du lecteur, ces références créent une rupture manifeste entre le discours savant et le discours profane mais aussi entre la lecture savante et la lecture profane. Cet ensemble de marqueurs de la «scientificité »du texte sont parfois rendus indispensables par le format des revues et des collections des maisons d'édition, proposant systématiquement une notice biographique en tête des articles ou sur la jaquette de couverture des livres. L'absence de ces références équivaut alors à une suspicion d'amateurisme qui nuit à la légitimité du propos. 
En tant que sociologue, l'auteur prétend avoir une vision synoptique des phénomènes sociaux dont il nous parle. A la différence de ces auteurs de roman qui décrivent une situation à travers le regard et les états de conscience d'un des personnages, l'auteur d'un texte de sociologie standard adopte un point de vue en surplomb ou bien adopte successivement différents points de vue, ce qu'aucun des agents étudiés ne sera jamais en état de faire. Ainsi, parler d'une grève «du point de vue du patronat » et «du point de vue des syndicats » est, pour le sociologue, à la fois une façon de persuader le lecteur que son propos est axiomatiquement neutre (ce qui n'est pas nécessairement le cas) et qu'il présente un intérêt irremplaçable, lui seul, de par sa profession, étant à même de rassembler des informations ordinairement séparées et de les articuler dans un même discours.

Pour S. R. Suleiman, l'effacement et la prise de distance du narrateur par rapport à l'histoire qu'il nous raconte ne sont pas des caractères pertinents pour l'identification d'un texte comme « roman à thèse ». Les textes relevant du genre peuvent être rédigés par un narrateur omniscient, extérieur au récit, du point de vue d'un des personnages (ou de plusieurs successivement) à la troisième personne ou même, à la première personne. Cependant, la formule prédominante dans ce genre est celle d'un narrateur extérieur à l'intrigue et disposant d'une vision synoptique sur l'action. C'est le cas dans le roman que S. R. Suleiman considère comme le plus typique du genre: L'Étape de Paul Bourget (1902).

L'Etape, reconnu dès sa parution comme un roman à thèse, a permis à Bourget «d'exprimer, par le truchement d'êtres fictifs, les principes qu'il considérait comme essentiels à une vie politique normale et saine » (Feuillerat, 1938).

Historiquement, L'Étape s'insère dans le climat intellectuel et politique de l'affaire Dreyfus. C'est un livre de combat des partisans de l'ordre, des antidreyfusards. L'Étape est l'histoire d'un jeune homme qui, d'anticlérical et socialiste, devient catholique et monarchiste. L'histoire se déroule en 1901. Jean Monneron, élevé d'après les principes «fanatiques », républicains et farouchement 
anticléricaux de Joseph Monneron son père, finit par renier ces principes et par épouser à la fois la fille et les idées de son ancien professeur, le «traditionaliste » Victor Ferrand. Celui-ci, ennemi des « faux dogmes de 1789 » est « un des chefs les plus en vue de la philosophie catholique dans l'Université »...

Jean Monneron, après quelques hésitations, devient un héros positif qui choisit la «bonne voie » et réussit dans la vie. Le roman aurait pu servir de démonstration aux mêmes thèses si le personnage, changé en héros négatif, avait choisi la «mauvaise voie » et connu l'échec et la déchéance.

Dans le roman, deux «supersystèmes idéologiques» s'affrontent, l'un est positif et l'autre négatif. Les deux questions que pose S.R. Suleiman sont: premièrement, comment cette valorisation inambiguë s'effectue et deuxièmement, par quels moyens (rhétoriques) s'impose-t-elle comme valable au lecteur?

Dans L'Étape, le rôle du narrateur est capital dans cette valorisation inambiguë. Il est omniscient, parle «avec la voix de la Vérité » et énonce explicitement des jugements qui confirment ou infirment les prises de position des personnages. Source de l'histoire qu'il raconte (comme le sociologue présentant ses données), le narrateur fait figure non seulement d'auteur mais aussi d'autorité. Puisque c'est sa voix qui nous informe des actions des personnages et des circonstances où celles-ci ont lieu, et puisque nous devons considérer - en vertu du pacte formel qui, dans le roman réaliste, lie le destinateur de l'histoire au destinataire - que ce que cette voix raconte est «vrai», il en résulte un effet de glissement qui fait que nous acceptons comme «vrai » non seulement ce que le narrateur nous dit des actions et des circonstances, mais aussi tout ce qu'il énonce comme jugement ou comme interprétation. Le narrateur devient ainsi non seulement source de l'histoire mais aussi interprète ultime du sens de celle-ci (Suleiman, 1983, p. 90).

La double fonction de source et d'interprète qui est celle de Paul Bourget dans L'Étape n'est-elle pas aussi celle de beaucoup d'auteurs de textes sociologiques? N'y a-t-il pas un parallèle étroit 
entre la révolte, toujours possible, d'un lecteur de roman à thèse, refusant le pacte formel, accusant le romancier d'avoir truqué son jeu, d'avoir fait du vraisemblable un procédé et le fréquent refus qu'opposent les profanes à l'autorité des textes sociologiques?

\section{Le texte sociologique conduit le lecteur d'une préconception inexacte ou fausse vers une représentation plus exacte ou plus juste de la réalité}

Selon Gusfield, l'écrit sociologique s'efforce de convaincre au moyen d'arguments enchaînés dans une rhétorique de la persuasion conduisant le lecteur de préjugés inexacts ou faux vers une représentation plus exacte ou plus juste de la réalité. Le plan de l'écrit sociologique satisfait typiquement cette exigence. Au départ, une revue de littérature permet de poser l'état actuel des connaissances sur le sujet. Ensuite vient un exposé des faits nouveaux avancés par le chercheur et un effort pour certifier la validité de ces faits par référence à une «méthode » parée du label de la scientificité. Enfin, la conclusion ajoute les savoirs nouveaux aux savoirs préexistants ou se substitue partiellement ou totalement à eux.

Chaque fois qu'un comité de lecture rejette un article «parce qu'il n'apporte rien de nouveau », il confirme la force de cet idéal qui fait obligation au chercheur (comme à l'artiste) de surprendre en produisant des résultats imprévus.

Le roman à thèse cherche « à démontrer une vérité », au double sens d'apporter un enseignement et d'apporter une preuve (Suleiman, 1983, p 90). Il partage cette caractéristique avec l'exemplum de l'ancienne rhétorique, la fable et la parabole. Comme les deux dernières, il lui arrive d'aller plus loin et d'exhorter le lecteur à rendre ses actions conformes à la vérité qu'il énonce... Mais n'est-ce pas très exactement ce que font les chercheurs en sciences sociales sommés de justifier de l'utilité sociale de leurs travaux, lorsque ce ne sont pas les fonctionnaires, les hommes politiques ou les journalistes qui tirent pour eux les «conclusions pratiques» de la vérité qu'ils énoncent? 
Dans un roman à thèse d'apprentissage (Bildungsroman), le lecteur est appelé soit à « répéter» l'évolution du sujet de l'histoire vers la « bonne » doctrine (variante exemplaire positive), soit à rejeter l'évolution du sujet au nom de cette doctrine (variante exemplaire négative). Dans un cas comme dans l'autre, le lecteur est censé accomplir une évolution idéologique, qui à la limite peut être une conversion. Ce modèle présuppose un lecteur qui au début serait non convaincu ou même hostile à la thèse du roman. Ce n'est qu'à propos d'un tel lecteur que l'on peut parler d'une véritable persuasion exercée par le roman. Il est évident cependant que les lecteurs déjà convaincus ne peuvent être exclus. Dans leur cas, la rhétorique de l'oeuvre fonctionne moins comme persuasion que comme confirmation: la lecture renforce l'adhésion du lecteur à la «bonne » doctrine, en lui fournissant l'occasion de juger les actions du protagoniste selon ses propres critères, qui sont aussi ceux du supersystème idéologique de l'oeuvre.

Un écrit sociologique standard est souvent construit comme une confirmation ou un démenti d'une thèse énoncé par un maître de la discipline ou, plus modestement, par le professeur ou le directeur du laboratoire. Le lecteur est supposé s’identifier aux chercheurs qui ont réalisé l'enquête (il pourrait même, en principe, éprouver le besoin de dupliquer l'enquête pour en vérifier les résultats). Les «faits observés » par le sociologue composent une histoire (ou une série de très brèves micro-histoires désignées, à la limite, par un code sur un tableau). ${ }^{3}$

Ils n'ont d'intérêt que s'ils orientent le lecteur vers une conclusion bien définie, confirmation ou démenti de ce « supersystème idéologique de référence » que constitue la théorie sociologique mise à l'épreuve par l'enquête.

Dans un roman à thèse, le héros subit toujours une ou plusieurs épreuves qui sont aussi des mises à l'épreuve de la thèse défendue par l'auteur. La notion d'épreuve comporte celle de confrontation ou de lutte: pour surmonter l'épreuve, le héros doit vaincre un adversaire. Or, le héros d'apprentissage est avant tout un être passif: c'est l'acquisition de la connaissance qui inaugurera, pour lui, l'ère de 
l'action. L'épreuve d'initiation «passive », où le héros n'agit pas (encore) est une épreuve d'interprétation. Le héros est « mis devant une situation (ou un texte) qu'il doit comprendre et expliquer. Surmonter l'épreuve, ce n'est rien de plus - mais rien de moins - que de découvrir le sens, donner la bonne interprétation »... (p. 68). La conception de l'interprétation qui sous-tend le roman à thèse aboutit non pas à la prise de conscience de la vulnérabilité de toute interprétation, mais à son contraire: l'épreuve d'interprétation mène ici à l'affirmation d'un savoir « vrai » sans réserve.

\section{Le texte sociologique s'adresse à un lecteur « indéterminé » et ne fait jamais appel, pour convaincre, à une caractéristique particulière d'un lecteur supposé}

Cette exigence est conforme à l'idéal universaliste de la science mais elle a évidemment le caractère d'une fiction ou d'un artifice rhétorique. Comme on sait, les articles de Sociologie sont rédigés prioritairement en fonction des exigences - parfois très strictes - des comités de rédaction des revues (comme on peut le vérifier en comparant des articles d'un même auteur publiés dans différentes revues). Les rapports de recherche sont soumis à la logique des appels d'offre des commanditaires et, plus généralement, aux impératifs diplomatiques des relations entre les équipes de recherche, les organismes de recherche et les agences publiques ou privées qui les financent. Les initiés et les membres de la profession perçoivent bien ces contraintes de forme (et parfois de fond) mais elles ne sont pas signalées dans le texte. Le lecteur supposé est un membre quelconque et indéfini de l'espèce humaine même si, en pratique, le style d'écriture, le vocabulaire, les allusions savantes en réservent la pleine compréhension à un public de spécialistes.

Sur ce plan, le roman à thèse se distingue peu de l'écrit sociologique: lui aussi s'adresse formellement à un lecteur indéfini et pratiquement, à une «clientèle» de lecteur que le texte se garde bien de définir explicitement. L'Enfance d'un Chef de J. P. Sartre offre un cas intéressant de texte formellement adressé à n'importe 
quel lecteur mais supposant, en fait, pour être compris, un lecteur spécifique. On y voit un jeune héros, Lucien, apprendre à devenir « un homme », c'est à dire un antisémite, un être qui se sent « dur et lourd comme une pierre », un être qui se fixe pour maxime « de ne pas chercher à voir en soi, il n'y a pas d'erreur plus dangereuse », mais c'est la parodie d'un roman à thèse «de droite », tel qu'aurait pu l'écrire Paul Bourget. Elle doit être lue sur un mode ironique, comme une antiphrase. Cependant, rien ne signale dans le texte que cette lecture est « la bonne ». La solution suppose connus les autres écrits et les choix éthico-politiques de J. P. Sartre.

\section{Le sociologue s'efforce de convaincre son lecteur que ses assertions sur la réalité ne sont pas les produits de son imagination mais le résultat d'une observation méthodique}

Selon Joe Gusfield, le sociologue est animé d'un idéal voisin du romancier « réaliste» mais inverse de la plupart des auteurs littéraires, qui considèrent comme une performance positive la rédaction d'une histoire très éloignée de leur expérience vécue. Le sociologue s'efforce toujours de montrer qu'il n'a rien inventé. Un des procédés les plus fréquemment employés consiste à faire parler un collègue - dont on cite les travaux - ou mieux un grand ancêtre. D'autres procédés consistent à faire répondre un échantillon représentatif de personnes enquêtées à une question, ou à insérer des extraits d'interviews dans le corps d'une démonstration, de telle sorte que ce soit les personnes enquêtées elles-mêmes qui disent ce que le sociologue hésite à énoncer par lui-même. Réponses quantifiées à une question, extraits d'entretiens, comptes rendus d'observations, fac-similés de documents jouent le rôle de preuves à l'appui mais aussi, inévitablement, de leurre: puisque ce n'est pas le sociologue qui le dit, pourquoi le soupçonner de l'avoir inventé?

Dans le roman à thèse, il arrive fréquemment que le narrateur s'abstienne de tout jugement de valeur sur les événements et les faits et gestes des personnages, laissant à ceux-ci le soin de dire au lecteur ce qu'il faut penser de la situation romanesque et comment elle 
confirme ou infirme les principes doctrinaux qu'elle illustre. Ainsi, dans L'Etape, plusieurs personnages prennent la parole pour apprendre à Jean, le héros (et indirectement au lecteur) ce qu'il faut penser de Joseph Monneron son père. On apprend ainsi que Joseph est un « déclassé », que sa carrière montre la « complicité de l'Etat, tel que la Révolution nous l'a fait », que «son irréligion est comme son radicalisme, la preuve qu'il ne vit pas avec ses morts », qu'il est « orgueilleux », qu'il « a rompu avec la tradition de sa race », que « c'est un honnête homme avec les idées d'un sectaire »... Tous ces propos indigènes viennent confirmer et redoubler les commentaires que le narrateur omniscient adresse directement au lecteur tels que: « il est fier », il est « un exemplaire absolu du Jacobin », il est «hors de toute espèce de religion »... Comme le narrateur s'exprime généralement avec plus de retenue que les personnages qu'il fait parler, on pourrait dire, en restant strictement dans le cadre de la fiction, que « comme un sociologue », il n'a rien inventé.

Ainsi donc, si l'on s'en tient au texte, sans faire de supposition sur la validité des méthodes d'investigation sociologique, l'écrit sociologique et le roman à thèse apparaissent tous deux comme des genres réalistes et se distinguent nettement d'autres genres comme l'allégorie religieuse ou politique (Pilgrim's Progress, L'Ile des Pingouins), ou le conte philosophique (Candide) qui, pour partager la vocation didactique et persuasive du roman à thèse et de la thèse sociologique, s'en différencient par leur statut de textes non réalistes.

\section{Le sociologue s'efforce, au fil de son texte, de passer de définitions confuses et ambiguës à des définitions claires et certaines}

Comme le signale Joe Gusfield, l'orientation du texte sociologique vers un résultat clair, simple et certain est considérablement amplifiée lors de la restitution du texte par des enseignants, des étudiants, des journalistes ou des hommes politiques qui, non contents de ne retenir que « l'essentiel» transforment souvent en statistique vraie un chiffre conjectural et une simple thèse en vérité officielle. 
Le passage du complexe au simple, du confus au clair, de l'obscur au lumineux n'est pas toujours évident dans les écrits des sociologues mais ce sont incontestablement les qualités qui sont louées dans un texte, qui font son succés. Un des procédés les plus fréquents consiste à ramener une multiplicité de variables explicatives à un facteur unique et déterminant.

En tant que texte savant, l'écrit sociologique est fait pour être résumé et cité jusqu'à terminer comme note de bas de page d'un écrit abstrait (et souvent, une chaîne de citations intermédiaires s'interpose entre le texte original et le résumé final). A la limite, la mention du titre, de l'auteur, de la date de publication et de l'éditeur vaut comme preuve d'une thèse condensée en une seule phrase. Sous ce rapport, l'écrit sociologique est très peu spécifique. Tout roman, toute oeuvre de fiction se prêtent à une lecture «à thèse ». Il est toujours possible d'en extraire une maxime ayant une portée générale. On peut même dire que toute histoire racontée par quelqu'un à un auditoire doit justifier sa propre existence (et l'acte même de la raconter) en répondant à la question: qu'est-ce que cela prouve? («Qu'est-ce que vous essayez de dire en racontant cette histoire?»). Au XVII ${ }^{\mathrm{e}}$ siècle, les gens de lettres appréciaient beaucoup Le traité du poème épique de P. Le Bossu, qui voyait en L'Illiade une illustration de la maxime: « la mésintelligence des princes ruine leurs propres Etats », tandis que L'Odyssée était censée démontrer que « l'absence d'une personne hors de chez soi, ou qui n'a point l'œil à ce qui s'y fait, y cause de grands désordres » (Kibédi-Varga, 1976).

Si le texte sociologique - comme tous les textes scientifiques - est fait pour être cité, au Moyen Age, l'exemplum sous toutes ses formes - mais tout particulièrement sous sa forme fictive - fut largement utilisé par les prédicateurs qui cherchaient à inculquer les doctrines de l'église et les principes moraux chrétiens à leurs auditoires, en leur racontant des histoires. Les monographies sociologiques sont résumées et rassemblées dans des ouvrages de synthèses et dans des manuels (et toute particulièrement aux EtatsUnis). De même, des recueils d'exemples circulaient dès le XIII ${ }^{\mathrm{e}}$ siècle et les prédicateurs à court d'inspiration pouvaient y puiser la matière de leurs discours. 


\section{Le sociologue explique le comportement des agents par des influences extérieures et tend à minimiser le rôle des caractéristiques internes (passions, personnalité, volonté, intelligence...)}

Typiquement, un sociologue explique les comportements individuels par des éléments de la structure sociale qui les « déterminent » ou leur « donnent sens ». Lorsque des facteurs internes à l'individu (comme l'humeur ou la volonté) sont évoqués, c'est souvent pour en rechercher l'origine dans le passé de l'agent et, à nouveau, dans des facteurs d'environnement. La pratique qui consiste à expliquer les variables «dépendantes » telles que l'opinion politique par des variables «explicatives » telles que la profession du père ou la profession exercée illustre ce point, tout comme la thèse sous-jacente de Goffman dans Asile (les fous se conforment à un rôle prévu pour eux par l'organisation asilaire et développent une personnalité partiellement autonome et souvent quasi-normale dans les marges et les trous de cette organisation).

Pour ce qui concerne le roman à thèse, on peut revenir à l'analyse que fait $S$. R. Suleiman de l'entrée en scène de Joseph Monneron dans L'Etape (p. 211-213). Dans les deux premiers chapitres du livre, et avant qu'il n'ait fait une seule apparition « en chair et en os », les futurs fait et gestes de Joseph Monneron sont expliqués par un ensemble de "variables explicatives » quasisociologiques. Ainsi, le narrateur omniscient et divers personnages du roman nous apprennent que Joseph Monneron est « professeur de lycée », «fils de cultivateur », «fils de Jacobin », « irréligieux », «radical », «séparé de sa famille dès l'enfance», «Dreyfusard ». C'est un produit du système démocratique, dont «l'esprit est de niveler les classes, d'égaliser pour tous le point de départ, de faciliter à l'individu les ascensions immédiates »... Autant d'explications anticipées (et par le contexte) de ce qui va se passer par la suite. Tous ces renseignements biographiques sont accompagnés d'interprétations et de jugements de valeurs énoncés par le narrateur - qui dit la vérité par définition - et par des personnages déjà constitués comme des interprètes valables et autorisés. Quant à Jean Monneron, il n'est pas 
donné d'emblée comme interprète valable de ce qu'il vit. Il ne peut y avoir aucun doute sur la valorisation négative de ses commentaires de «professeur chimérique, volontairement aveugle sur des vérités trop pénibles $» . .$.

Ainsi donc, l'auteur du roman à thèse, tout comme l'auteur d'un écrit sociologique standard, tend à disqualifier les explications «internes»données par les agents eux-mêmes sur leur propre comportement et à privilégier les explications par des facteurs extérieurs et données de l'extérieur. Ces explications sont fournies par le narrateur omniscient et redoublées ou confirmées par des personnages du roman (ou par des personnes interrogées par le sociologue).

\section{Où sont les différences entre romans à thèse et thèses de Sociologie?}

Si l'on s'en tient strictement à l'étude de la relation entre le texte et le lecteur, on ne peut qu'être frappé des correspondances entre un genre réputé "littéraire" et un autre réputé "scientifique": auteur distant, hors de son propre texte mais produisant des effets d'autorité; utilisation des personnages (des enquêtés) pour dire au lecteur ce qu'on ne pourrait dire soi-même sans se discréditer; mouvement rhétorique conduisant le lecteur, à travers un certain nombre d'épreuves, d'une réalité confuse et mal connue vers une représentation plus simple, claire et certaine; dissimulation du lecteur auquel on s'adresse derrière l'écran d'un lecteur idéal et impersonnel; traitement formel différencié des "faits" et des "interprétations"; explication des comportements humains par des forces externes déterminantes...

Pour restaurer, du point de vue du lecteur, une différence entre thèse de sociologie et roman à thèse, il faut, comme y invitent souvent les textes, faire du «hors-texte», en revenir aux sources et aux débats

La lecture "savante" découle d'un apprentissage, suppose un temps de lecture long et un appareillage érudit: bibliothèques 
spécialisées, colloques et débats, revues comportant des notes critiques... Tous ces moyens sont utiles mais peu opérants lorsqu'il s'agit de contrôler la fidélité d'une narration à la situation dont elle prétend rendre compte. Pour que l'adéquation au réel du propos se trouve mise à l'épreuve (et non pas sa vraisemblance compte tenu de ce que l'on croit savoir), il faudrait que l'auteur «sociologue » constitue, en dehors et à côté de la rédaction de sa « thèse » un corpus documentaire méthodique, disponible en un lieu répertorié (qui peut être une mémoire d'ordinateur). Il faudrait aussi qu' au moins quelques lecteurs, « sociologues professionnels », prennent la peine et le temps de vérifier par sondage au moins quelques éléments de la narration publiée, pour remonter aux sources et vérifier que la base documentaire existe et qu'elle a été fidèlement respectée, dans l'esprit si non dans la lettre. Ainsi pourrait-on faire le départage entre un dialogue inventé et un dialogue fidèlement transcrit, entre une scène fictive et une réunion qui a effectivement eu lieu et dont on retrouverait dans les archives les comptes rendus dactylographiés (par un rédacteur qui n'était pas le sociologue), les notes inscrites en cours de réunion par l'animateur sur son tableau de papier, la copie des transparents projetés sur l'écran du rétroprojecteur, et peut être même quelques notes manuscrites prises par les participants. A propos d'un différent entre collègues de travail, ou pourrait conserver sur papier ou sur une mémoire informatique la série complète des @mail échangés entre les protagonistes, etc.

Procéder à de tels contrôles de qualité de la production sociologique n'est pas dans les moeurs. L'usage veut que l'on discute d'un point de vue «théorique » ou «méthodologique » les faits rapportés, sans les vérifier. C'est peut-être dommage.

Un contrôle de la base documentaire montrerait à n'en pas douter la différence entre une thèse de sociologie, lorsqu' elle est bien faite, et un simple roman à thèse ou une simple enquête journalistique, fondée seulement sur des témoignages verbaux.

Encore faudrait-il que la travail de constitution d'archives de terrain soit encouragé et valorisé et que l'examen de ce travail par des collègues soit considéré comme légitime, utile, et même 
nécessaire. Encore faudrait-il qu'il soit systématiquement réalisé, par exemple par les jury de thèse ou les lecteurs d'articles soumis dans les revues. Encore faudrait-il qu'une sorte de «label» de fidélité aux sources puisse être décerné lorsque les tests ont donné des résultats convaincants.

Le risque de telles pratiques est évidemment de conduire à un terrorisme de la preuve, au formalisme de la citation « à la lettre ». C'est me semble-t-il le prix à payer pour justifier la garantie d'un travail que l'on veut aussi «scientifique» que possible. Je vois un autre avantage dans la séparation entre la rédaction de la thèse proprement dit et le corpus documentaire de référence: il permet l'allégement de la rédaction finale et une mise en récit à mon avis indispensable aujourd'hui, pour que la sociologie reste accessible au public des non-spécialistes.

\section{Conclusion}

Une raisonnement trop rapide pourrait porter à conclure que "l'autorité scientifique" n'est qu'un cas particulier de l'autorité fictive. La lecture ordinaire d'une thèse de Sociologie s'apparente en effet à la lecture d'un roman à thèse, mais il faut aller plus loin et montrer que la fabrication d'une thèse de Sociologie de qualité n'est pas la même chose que la fabrication d'un roman à thèse.

Cette différence dans le modus operandi ne s'applique sans doute pas à toute la production sociologique. Bien des exemples plaident en faveur de la confusion des genres (roman à thèse, journalisme d'investigation, essai de philosophie sociale et thèse de Sociologie). Trop souvent, les étudiants en Sociologie partent de préconceptions tirées de lectures savantes et cherchent des observations qui viendront confirmer ce qu'ils veulent démontrer. Ils citent, mais c'est pour flatter et par ordre de préséance, etc.

Ces critiques peuvent être adressées aux enquêtes quantitatives dont la mise en oeuvre suppose la formulation à priori d'hypothèses et le déploiement d'une méthodologie qui prédéfinit étroitement les 
questions auxquelles on cherche une réponse et la manière dont les réponses seront obtenues.

Elle s'applique aussi aux monographies de terrain, dès l'ors que l'exigence de contrôle des sources est relâchée. Il me semble en effet qu'on peut considérer une monographie de terrain comme un roman d'initiation dans lequel le rôle du héros serait tenu par le chercheur, découvrant un milieu en même temps qu'il le fait découvrir à ses lecteurs et passant, au cours d'une série d'épreuves, de l'ignorance à la connaissance. La tension entre les croyances propres au milieu étudié et les croyances académiques au nom desquelles la recherche est entreprise donne une dimension antagonique au récit. La fidélité du compte rendu aux expériences du chercheur, en dépit des difficultés et des risques inhérents au dévoilement publique des rapports sociaux observés est une de ces épreuves par lesquelles le chercheur-héros doit prouver son attachement à l'idéal savant contre les idéaux indigènes pour mériter son élection au sein de la communauté scientifique.

Face à la tentation scientiste de protéger les démarches savantes de toute ressemblance avec les fictions littéraires, on peut se demander si ce n'est pas au contraire en poussant jusqu' au point limite le schéma du roman d'initiation, en faisant des épreuves de véritables épreuves de vérité, et des soi-disant "observations de terrain" de véritables expérimentations, (au cours desquelles le chercheur prend le risque de l'échec), qu' on peut échapper aux limites étroites du récit "à thèse" qui ne fait jamais qu'illustrer les certitudes acquises, ("scientifiques" ou autre), au lieu de les tester pour les dépasser.

\section{Notas}

1 Le roman à thèse est souvent considéré comme un genre indigne, qui n'ose pas dire son nom. C'est toujours le roman d'un autre. On dit qu'il trahit la littérature parce que: "Pour soutenir la thèse, il faut truquer les personnages et les situations et forcer, partialiser l'observation" ( Enquête sur le mouvement littéraire, de 1904, citée dans Charles Brun, Le Roman social en France au XIX siècle, V. Giard et E. Biäre, 1910, p. 59, 60). 
2 Subdivisé en deux sous-genres, roman d'initiation (Bildungsroman) et roman antagonique, le roman à thèse est lui même inclus dans deux supergenres: le récit exemplaire (où il côtoye l'exemplum de l'ancienne rhétorique, les fables, les paraboles) et le roman réaliste, où l'on trouverait, par exemple, des romans de Balzac, Zola ou Maupassant.

3 La définition minimale d'une histoire, qui peut tenir en une phrase, comporte un sujet et une transformation affectant ce sujet à travers le temps.

Resumo: Serão as marcas retóricas de "cientificidade" do discurso sociológico específicas? Para abordar esta questão sensível, partimos de um quadro de procedimentos retóricos correntemente utilizados em Sociologia. Deduzimos as características gerais do gênero "escrito sociológico". Comparamos este gênero com um gênero literário às vezes próximo e às vezes distinto: roman à these (um gênero menor, bastante praticado na França no início do século XX e ilustrado, notadamente, por Charles Mauras e Paul Bourget). Os paralelos são desconcertantes. Além disso, se as diferenças entre a tese sociológica e o roman à these não são marcadas no texto, então o que eventualmente caracteriza sua "natureza científica"? Talvez sejam as frágeis condições de produção e recepção do conhecimento: no debate e crítica das fontes se efetivamente implementado. Mas será sempre o caso?

Palavras-chave: Sociologia, roman à these, gênero literário, retórica, cientificidade.

Abstract: Are the rhetorical marks in sociological discourse specific and of a "scientific" nature? To tackle this delicate question, let us start by drawing up the generic characteristics of sociological texts. Let us compare these characteristics with those of a literary genre: the French "roman à these" from the beginning of the XX century. This old-fashioned and decried litterature will serve as a driving bolt in order to demonstrate that a formalism sometimes asserted as scientific, does not guarantee scientificity, but just conformity to editorial requirement. To conclude, if the difference between sociologal theses and novel is not embedded in the text itself then, what eventually makes its "scientific nature"? Maybe just the fragile conditions of its production and reception: debate and criticism of the sources, if effectively implemented. But is this always the case?

Key-words: Sociology, novel, literary genre, rhetoric, scientificity. 


\section{Liste des références}

BARRÈS, Maurice. Les déracinés. Ed. orig. Paris: Hachette, 1897.

BLAU, Peter M., DUNCAN, Otis D. The American occupational structure. New York: John Wiley and Sons, 1967.

BOURDIEU, Pierre. La distinction. Paris: Editions de Minuit, 1979.

BOURGET, Paul. L'Étape. Ed. orig. 2 tomes. Paris: Plon, 1902.

FEUILLERAT, A. Paul Bourget: histoire d'un esprit sous la troisième République. Paris: Plon, 1938.

GOFFMAN, Erving. Les rites d'interaction. Paris: Editions de Minuit, 1974.

GUSFIELD, Joe. Sociology as a Litteracy genre.Conférence prononcée à l'EHESS à Paris, le 24 mars 1987.

KIBÉDI-VARGA, A. L'invention de la fable. Poétique, n. 25, 1976.

LATOUR, Bruno; WOOLGAR, S. Laboratory life: the Social Construction of Scientific Facts. Los Angeles: Sage, 1979.

LATOUR, Bruno; FABRI, Paolo. Pouvoir et devoir dans un article de Sciences Exactes. Actes de la Recherche en Sciences Sociales, n.13, p. 81-95, 1977.

NIZAN, Paul. La Conspiration. Ed. orig. Paris: Gallimard, 1938.

SARTRE, Jean-Paul. L'Enfance d'un Chef. In: . Le Mur. Ed. orig. Paris, Gallimard, 1939.

SCHELLING, Thomas C. La tyrannie des petites décisions. Paris: PUF, 1980.

SULEIMAN, Susan Rubin. Le Roman à Thèse. Paris: Presse Universitaire de France, 1983.

WEBER, Max. Economie et Société. Paris: Plon, 1971.

WHYTE, William Foote. Street Corner Society: the social structure of an Italian slum. Chicago: The University of Chicago Press, 1943, 1965. 